\title{
Diffraction-based phase calibration of spatial light modulators with binary phase Fresnel lenses
}

\author{
Omel Mendoza-Yero, Gladys Mínguez-Vega, Lluís Martínez-León, Miguel Carbonell-Leal, \\ Mercedes Fernández-Alonso, Carlos Doñate-Buendía, Jorge Pérez-Vizcaíno, and Jesús Lancis \\ GROC·UJI, Institute of New Imaging Technologies, Universitat Jaume I, 12071-Castelló, Spain
}

\begin{abstract}
We propose a simple and robust method to determine the calibration function of phase-only spatial light modulators (SLMs). The proposed method is based on the codification of binary phase Fresnel lenses (BPFLs) onto an SLM. At the principal focal plane of a BPFL, the focal irradiance is collected with a single device just able to measure intensitydependent signals, e.g., CCD camera, photodiodes, power meter, etc. In accordance with the theoretical model, it is easy to extract the desired calibration function from the numerical processing of the experimental data. The lack of an interferometric optical arrangement, and the use of minimal optical components allow a fast alignment of the setup, which is in fact poorly dependent on environmental fluctuations. In addition, the effects of the zeroorder, commonly presented in the diffraction-based methods, are drastically reduced because measurements are carried out only in the vicinity of the focal points, where main light contributions are coming from diffracted light at the BPFL. Furthermore, owing to the simplicity of the method, full calibration can be done, in most practical situations, without moving the SLM from the original place for a given application.
\end{abstract}

Index Terms - Spatial light modulator, calibration, diffractive optical elements.

\section{INTRODUCTION}

Spatial light modulators can be regarded as excellent tools to manipulate at will the amplitude and phase of laser beams. They have been widely used to encode diffractive optical elements (DOEs) and to manipulate a variety of light properties, with important roles in linear/nonlinear microscopy [1], micro-processing of materials [2], spatial beam shaping and optical tweezers [3], wavefront sensors and adaptive optics [4], or pulse shaping [5], among many other applications.

Manuscript received October 1, 2015. This work was supported in part from MINECO (grant FIS2013-40666-P), Generalitat Valenciana (grants PROMETEO2012-021 and ISIC 2012/013), and Universitat Jaume I (P11B2012-55)

Omel Mendoza-Yero, Gladys Mínguez-Vega, Lluís Martínez-León, Miguel Carbonell-Leal, Carlos Doñate-Buendía, Jorge Pérez-Vizcaíno, and Jesús Lancis are with the Institute of New Imaging Technologies, Universitat Jaume I, 12071-Castelló, Spain (e-mail: omendoza@uji.es).
At present, spatial light modulation can be carried out with the help of different devices. For instance, by using parallel aligned liquid crystal on silicon (LCoS) SLMs, with refresh rates in the order of few tens of $\mathrm{Hz}$ and phase-only modulation mode, the required dynamic range of most applications is reached [6]. Other devices, such as the digital micromirrors devices (DMDs), with refresh rates up to tens of $\mathrm{kHz}$ and amplitude-modulation mode, may approach real-time responses [7]. In addition, deformable mirrors offer the possibility to correct the wavefront of light beams. The calibration method proposed in this paper will be applied to phase-only SLMs, such as the commercially available parallel aligned LCoS modulators.

Former devices usually required complex calibration procedures. In the case of liquid crystal SLMs, complete calibration may consider the own SLM as a retarder-rotator system [8], which often exhibited a coupled phase and polarization modulation. In this context, theoretical expressions for the eigenvalues and the eigenvectors for twisted-nematic liquid-crystal displays as a function of the twist angle and the birefringence have been derived [9]. In this manuscript, authors also discuss techniques for achieving amplitude-only modulation, and coupled amplitude and phase modulation. Using another technique in which Jones matrices describing their polarization, a characterization of a reflective Holoeye LC-R 2500 SLM was also carried out [10], and applied in a holographic optical tweezers setup. Furthermore, a full characterization of an LCoS SLM, for phase-mostly modulation has been done, showing that polar decomposition of Muller matrices determines the polarization properties of the device [11].

The calibration process determines the phase response of liquid crystal SLMs as a function of a certain control parameter, e.g., the voltage signal applied to each pixel of the device. The relation between the output phase values and the input signal e.g., 256 grey levels contained in the displayed image, is the so-called calibration curve/function. The calibration process is a mandatory task before carrying out most practical implementation of SLMs within optical systems. Now, several types of calibration methods have been reported. In general, they can be separated into two main groups. One of them are the interferometric methods whereas, on the other part, we can find the diffractive-based ones. 
However, there are a remaining, but less widespread, set of calibration methods that cannot be included into the above groups.

The first group includes, but is not limited to, methods like the Young's fringes-derived phase characterization method [12]. In this case, the interference pattern is achieved by using a mask with two pinholes at the SLM plane, being the translation of the fringes at the Fourier plane affected by the phase modulation at each pinhole. Furthermore, the contrast of the fringe patterns depends on the similarity degree between the two pinholes. When a SLM shows nonuniform spatial response in its phase-versus-voltages curve, relatively complex interferometric setups are applied for calibration purposes. This is the case of the optical setup shown in [13], where a Michelson architecture, composed, among other elements, of two polarizers, a piezo mirror, a beam splitter, and a CCD camera. The position dependent phase response of a transmissive SLM is measured and corrected in [14] with a Mach-Zehnder interferometer, including optical elements like polarizing beam splitters or two-lens Kepler-type imaging optics. A method, based on a shear interferometer, able to simultaneously measure the amplitude and phase modulation of a twisted liquid crystal display, in a relative simple manner, is shown in [15]. Here, it should be noted that apart from the inherent drawbacks related to the alignment of interferometers, as well as their high sensitivity to mechanical vibrations or air turbulences, above-mentioned calibration setups required a large number of optical elements.

Instead of interferometric, diffractive-based methods can be alternatively implemented for obtaining the calibration curves. In general these methods rely on the analysis of the diffraction field due to the interaction of light with certain multi-phase DOEs, previously encoded into the SLM [16]. To get the calibration curve, they employ phase retrieval algorithms. Other approaches simply quantify one of the parameters of the corresponding DOE in the far field, such as the intensity of the diffraction orders created by a two-level grating [17] or the visibility of a Fresnel image pattern [18]. Diffraction-based methods are in principle less sensitive to environmental disturbances, but in contrast they may be affected by residual intensity modulation, and/or by the discrepancies introduced by the zero order of diffraction associated with the nondiffracted light.

Other types of calibration methods, not included into the above-mentioned groups, use polarization set-ups. Basically, to obtain the calibration function, intensity measurements along cross-polarizers are performed [14]. The first polarizer rotates the input beam polarization at $45^{\circ}$ with respect to the aligned axis of the liquid crystal molecules. Then, a second polarizer is set at $-45^{\circ}$ with respect to the input polarization plane. As phase variations are converted into intensity fluctuations/phase shifts for calibration purposes, above configuration is known as intensity modulation. A complete characterization of a liquid crystal SLM is possible with a system including additional polarization elements [8,19].

At this point, it is important to mention that accuracy of most phase characterization methods can be significantly influenced by unwanted physical phenomena associated with the performance of SLMs. For instance, it is well-known that temporal fluctuations of the liquid crystal molecular orientation as a function of time causes depolarization effects, deteriorating the diffraction efficiency of SLM [20]. In fact, when temporal fluctuations occur, the hypothesis of constant phase during measuring time is no longer valid. Another harmful effect that should originate e.g., non-linear phase modulation or coupled amplitude modulation, is related to the Fabry-Perot multiple-beam interference generated by the intrinsic layer structure of the liquid crystal device [21]. In this work, authors pointed out that, in cases of illumination of LCoS SLMs under angles other than normal incidence or/and phase modulation regimes higher than $2 \pi$, multiple reflection interference is increased. These drawbacks, as well as many other problems, including non-uniformities in the backplane [14] or fringing field effects [22], should be taken into account to a greater or lesser extent depending on the accurate measurements required for a given application. Furthermore, detailed studies of their consequences on some interferometric or diffraction-based phase calibration techniques have been reported [20]. Hence, the study of above-mentioned effects is beyond of the scope of the present work.

In this manuscript we introduce an extraordinary simple and compact diffractive-based method for calibrating the phase response of a liquid crystal phase-only SLM. It is based on the measurement of the focal irradiance of binary phase Fresnel lenses (BPFLs) encoded into a phase-only SLM. Owing to circular symmetry of BPFLs, the measurement of the focal irradiance is carried out on-axis, which is usually of great convenience. Additionally, the influence of the zero order on the focal irradiance measurements is quite mitigated because of the preponderant diffraction nature of the collected light. Note that, in comparison with a Fourier hologram that uses a lens to focus the whole incoming light (modulated or not), Fresnel holograms given by BPFLs originate diffraction orders for which contribution of non-modulated light is negligible. On the other hand, the proposed diffraction-based optical setup can be less affected by environmental fluctuations than other two-arms interferometric arrangements used for the same purpose, with the exception of methods based on in-line interferometric setups that do not suffer from vibrations. As our method needs only to record the irradiance of a well spatially localized focal point, in principle, there is no need to use a measuring device with spatial resolution. That is, light intensity changes at the focal point can be collected with single-pixel detectors, e.g., photodiodes, power meters or even common spectrometers. Hence, the optical system is composed, apart from the SLM itself, only of a beam splitter and a light intensity-dependent measuring device. Furthermore, owing to the fact that intensity measurements are relative our calibrating method is generally quite forgiving to non-uniformity irradiance profiles, especially slowly varying changes.

The content of the manuscript is organized as follows. In the first section, we develop the theoretical model that supports the phase calibration method. In the second section, a commercially available SLM is employed to demonstrate the usefulness of the proposed phase calibration method. In the 
third section, a comparison of the obtained results with a similar off-axis method, characterized by the use of binary phase gratings, is carried out. Finally, in the last section, the main conclusions of our work are presented.

\section{THEORY UNDERLYING THE CALIBRATION METHOD}

In this section, the theoretical basis of our method is demonstrated. In particular, we will show that the diffraction efficiency of a BPFL depends on the difference between its two constituent phase levels. A BPFL consists of a set of radically symmetric phase rings, which interchange their phase values between two possible levels $\phi_{1}$ and $\phi_{2}$. The rings are designed in such a way that optical path lengths of rays diffracted from adjacent zones differ by an integral multiple of a design wavelength $\lambda$. Within the framework of the paraxial approximation, the radius $r_{n}$ for the $n$-th zone is given by $r_{n}^{2} \approx 2 n \lambda f$ [23]. Hence, BPFLs are optical elements periodic in $r^{2}$, with period $r_{1}^{2}$, and transmittance function $t(r)$ described by the Fourier series [24],

$$
\begin{aligned}
& t(r)=\frac{\exp \left(i \phi_{1}\right)+\exp \left(i \phi_{2}\right)}{2}+ \\
& +\sum_{\substack{m=-\infty \\
m \text { odd }}}^{m=\infty} \frac{i\left[\exp \left(i \phi_{2}\right)-\exp \left(i \phi_{1}\right)\right]}{\pi m} \exp \left(\frac{i \pi m}{\lambda f} r^{2}\right)
\end{aligned}
$$

After comparing the phase given in Eq. (1), with the quadratic phase introduced by a well-known spherical lens, $\pi r^{2} /(\lambda f)$, one can realize that incident plane waves are diffracted in form of spherical waves with foci located at the axial positions $f_{m}=r_{1}^{2} /(2 m \lambda)$. From Eq. (1), it is also apparent that the intensity of each focus can be calculated through the squared modulus of the corresponding coefficient in the Fourier series.

Neglecting reflection losses, we define the diffraction efficiency of a BPFL as the ratio between the integrated irradiance in the focus, and that of whole incident light. In mathematical terms, diffraction efficiency $\eta_{m}$ yields

$$
\eta_{m}=\left|\frac{i\left[\exp \left(i \phi_{2}\right)-\exp \left(i \phi_{1}\right)\right]}{\pi m}\right|^{2}=\left(\frac{2}{\pi m}\right)^{2} \operatorname{sen}^{2}\left(\frac{\phi_{2}-\phi_{1}}{2}\right)
$$

From Eq. (2), it is apparent that the diffraction efficiency will depend on the selected focus, whereas the highest focal intensity (main focus) is obtained in the case $m=1$. In addition, the maximum focal efficiency is achieved when $\phi_{2}-\phi_{1}$ is equal to multiples of $\pi$. Equation (2) is the main theoretical result that supports our phase calibration method.

Owing to the fact that each gray level sent to the SLM is transformed into a given phase value, a complete set of BPFLs can be always constructed by selecting pairs of gray levels, e.g., the first one is zero and the second is set within the range from 1 to 255. In our implementation, the gray level 0 (corresponding to the phase value $\phi_{1}$ ) is fixed for all BPFLs. The combinations of the gray level 0 with the remaining ones, allow us to define 255 different BPFLs. Then, measurements of the integrated irradiance at a given focal plane, for the whole set of BPFLs is enough to obtain the experimental curve of the diffraction efficiency against gray level shifts. From the theory discussed here, it might be clear that above experimental curve is similar to the one predicted by Eq. (2), but now in terms of the phase shifts. To obtain the phase calibration curve e.g., the dependence of the gray levels with phase values, a suited fitting between experiment and theory is done.

\section{EXPERIMENTAL IMPLEMENTATION OF THE METHOD}

The phase calibration method proposed in this manuscript was tested by using the optical setup shown in Fig. 1. Before it impinges onto the beam splitter, the light beam emitted by a He-Ne laser (model 1135P from UNIPHASE, emitting at $632.8 \mathrm{~nm}$, and maximum output power of $10 \mathrm{~mW}$ ) is conveniently expanded with a commercial telescope (model EL-25-5X-B from THORLABS). This allows filling the whole area of the SLM display (Reflective PLUTO Phase Only SLM from HOLOEYE) which has a resolution of $1920 \times 1080$ pixels, with 8 micrometers of pixel pitch. The light beam reflected from the SLM is then transmitted through the beam splitter and sent to a conventional CCD camera (model UI3480CP-M-GL from UEYE). Here, the use of a measuring device with spatial resolution is only motivated by the comparison (in the next section) of our method with an already established one. The intensity of the light at the CCD plane is always kept under the saturation level of the camera thanks to a previous attenuation of the light with neutral filters (not shown in the optical setup). In left bottom part of Fig. 1, the shape of a BPFL is also shown as an inset.

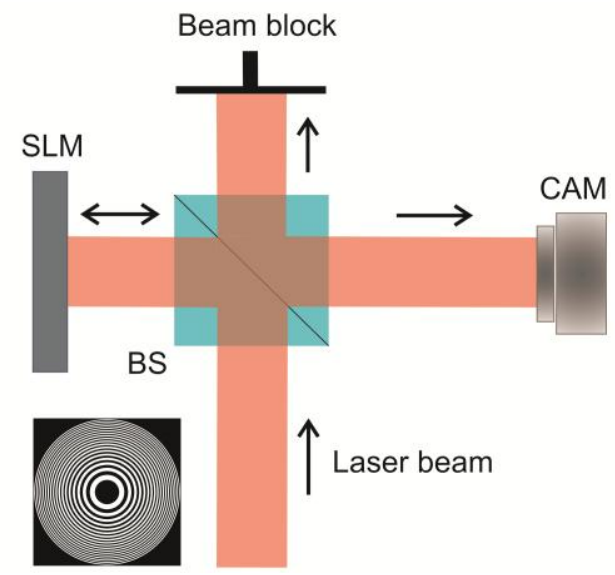

Fig. 1. Optical setup used for phase calibration of a phase-only SLM. The light from a He-Ne laser impinges onto the SLM after being reflected in the beam splitter. The camera collected the diffracted light at the focal plane of BPFLs encoded into the SLM.

We encode a set of 255 different BPFLs into the SLM, constructed following the criteria described in the previous section. In our experiment, the position of the camera coincides with the main focal plane of BPFLs, which is located at $300 \mathrm{~mm}$ from the SLM plane. The focal images due to the diffraction of light by BPFLs are recorded with the CCD camera. The experiment is controlled with a Matlab software that takes less than 5 minutes to collect and process the data. We realize that this time duration is mainly limited by the 
refresh rate of the SLM (up to $60 \mathrm{~Hz}$ ), and the integration time set in the camera, whereas in less extent by the computer hardware.

From the recorded images, it can be obtained a signal proportional to the integrated irradiance of each focus. Hence, we sum the intensity of all pixels contained within the focal spot of the images. The results are normalized and shown in Fig. 2(a). (a)

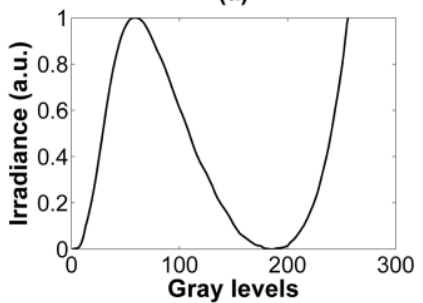

(b)

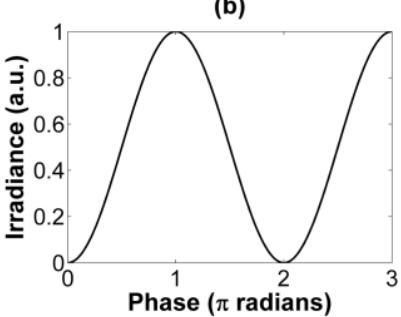

Fig. 2. Integrated irradiance at main focus plane of BPFLs in the region defined by the $1 / e^{2}$ criterion, a) experiment and $\mathrm{b}$ ) theoretical expression given in Eq. (2).

As the focal widths do not change when varying the phase of the BPFLs, both the number of pixels used to define the focal regions, and their locations are always the same. The focal region is defined by the $1 / e^{2}$ criterion for the beam width. The experimental curve of Fig. 2(a) shows a normalized integrated irradiance (or diffraction efficiency) behavior similar to the one described by Eq. (2), and represented in Fig. 2(b). Notice that, in Fig. 2(a) the irradiance depends on the different gray levels used to encoded the BPFLs, whereas in Fig. 2(b) the irradiance varies with the phase values. The discrepancies between both curves will allow us to extract the information regarding the phase calibration. Here it should be mentioned that some extreme points in the original experimental curve did not coincide with the values 0 and/or 1. This usually occurs, among other factors, due to nonhomogeneities in the spatial phase response of the SLM or in the linear polarization of the laser beam. By using a discrete uniform distribution, we simulate the effect of non-uniform phase encoding of BPFLs on the integrated irradiance. Our simulation reproduced what happened in the experiment with a root mean square error less than $14 \%$. Therefore, to obtain the experimental curve shown in Fig. 2(a), the original raw data curve was numerically processed, following a standard procedure e.g., see section 5.2 of manuscript [25].

From the results of Fig. 2 one can determine the phase calibration curve of the SLM, that is, the dependence of phase values with the gray levels. To do that, phase shifts in Eq. (2) are expressed as a function of the normalized irradiance values. Then, normalized irradiance values, now taken from the experimental curve, are substituted into Eq. (2). Owing to the fact that the gray levels and phase values are linked by a common irradiance value, the phase calibration function can be generated. Note that, to get a real function the above process is carried out separately for each section of the curve defined between two local minima and maxima or vice versa. The phase calibration function is shown in Fig. 3. From this figure, it is clear that phase range of the SLM is extended approximately from 0 to $3 \pi$. The information extracted from Fig. (3) can be useful for conveniently changing the look-up table, for example, to get a linear dependence between phase values and gray levels.

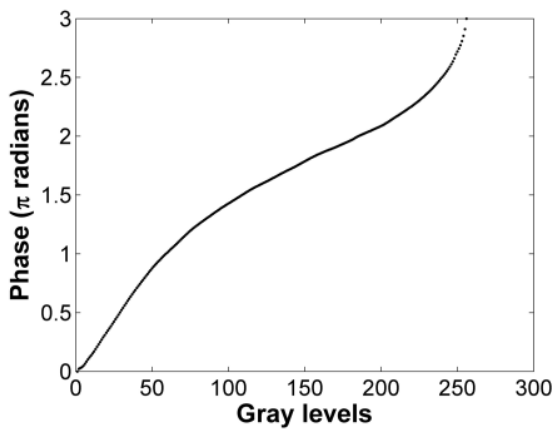

Fig. 3. Phase calibration function obtained with the proposed method, which is based on a set of BPFLs encoded into the SLM.

\section{VALIDATION OF THE METHOD AND DISCUSSIONS}

In this section we use a well-established phase calibration method [17] to validate our proposal. For the experiment, we took advantage of the optical setup described in Fig. (1), including the same optical components. However, this time instead of BPFLs, a set of 255 Ronchi grating patterns are encoded into the SLM. They were designed with the equal gray level distribution as used before for BPFLs. The corresponding diffraction pattern at the Fourier plane is recorded again with the CCD camera, but including now an extra refractive lens of $380 \mathrm{~mm}$ focal length. The integrated irradiance was measured and numerically processed [25] for all diffraction gratings. To define the integration region, the $1 / e^{2}$ criterion for the beam width is applied. Then, following a similar procedure as being described in section III, the phase calibration function is determined. In Fig. 4, this curve is represented with thick solid lines. In addition, with square symbols, but using only 33 points, is plotted again corresponding phase calibration curve obtained with our method, i.e., equal to the one shown in Fig. 3.

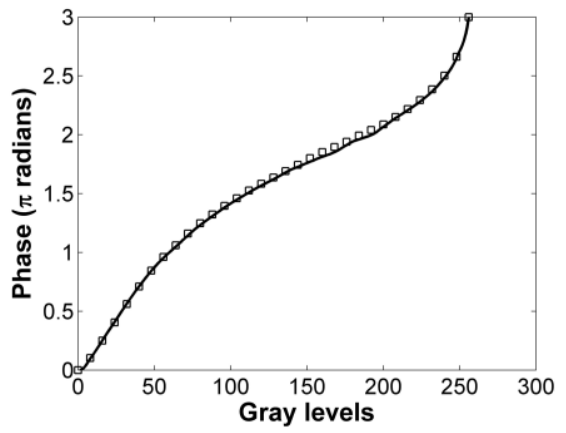

Fig. 4. Comparison between two phase calibration functions, a) curve plotted with thick lines, obtained with the Ronchi grating method introduced in [17], b) curve plotted with square symbols, obtained with our proposed method using BPFLs.

After a visual inspection of Fig. 4 one can realize that phase calibration functions achieved with both methods are very similar. The root mean square error yields $6.2 \%$. Small discrepancies between both results can arise due to fluctuations in the laser beam position and/or energy, as well as differences caused by the spatial shape of focal points. 
The last aspect may be better understood with the help of Fig. 5 , where typical foci recorded with both methods are shown. In the method based on the implementation of Ronchi gratings, the focus is off-axis, because one looks for the first diffraction order of each grating, which typically have non circular symmetric spatial shape. In contrast, by using the method based on BPFLs, all axial diffraction orders (or foci) are on-axis, and consequently approach better to a circular symmetric pattern. Hence, the selection of the integration region may be slightly affected by the spatial shape of the focus.
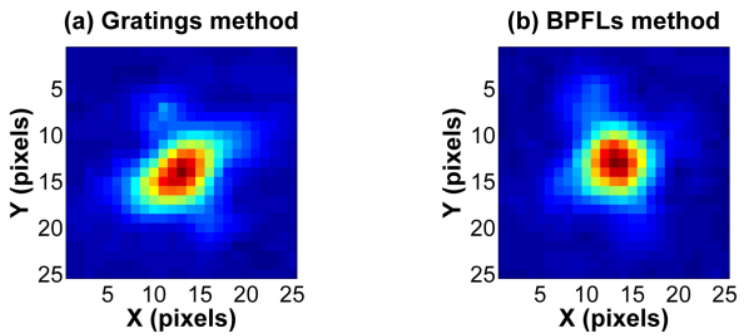

Fig. 5. Focal irradiance distribution of a pair of foci used for phase calibration, a) with the method based on Ronchi gratings [17], b) with the method based on BPFLs proposed in this manuscript.

So, from curves shown in Fig. (4) it is clear that our method offers reliable data for phase calibration purposes. In the laboratory, it has been also tested for different design parameters and conditions. In particular, for other periods of BPFLs, for measurements of the integrated irradiance at secondary focal planes, and for additional criteria regarding the definition of the integration region around a given focus. In all cases, the experiment gives results poorly dependent on the variable analyzed, with root mean square errors always less than $5 \%$. On the other hand, by means of numerical simulations we also tested the method for laser beams with Gaussian, and in general arbitrary, amplitude distributions at the SLM plane. The simulations basically show that phase calibration function does not change when modifying the amplitude of laser beams at the SLM plane. This conclusion might be expected since, from a practical point of view, relative and not absolute irradiance measurements are carried out.

The proposed method is mainly limited by the following aspects. We cannot correctly encode BPFLs with a main focal plane located below certain distance from the SLM. This critical distance can be calculated from the sampling criterion used to encode the minimum feature size of BPFLs. In our case, we assume that minimum feature size will be encoded with at least two pixels, which let us to a critical distance of approximately $150 \mathrm{~mm}$. Additionally, the proposed calibration method can give only a global value of the calibration function, which is unable to detect irregular phase responses associated with unexpected pixel behaviors or a non-uniform linear polarization of the laser beams, among other factors.

However, we believe that above-mentioned restrictions do not introduce severe problems in most applications, ranging from beam shaping to material processing or optical microscopy, being perhaps more critical for those situations where high quality spatial or temporal resolutions are needed. In these cases, the use of an SLM with increased spatial resolution, or the implementation of arrays of BPFLs may soften limitations.

\section{CONCLUSIONS}

In this manuscript, we propose an extremely compact phase calibration method based on irradiance measurements of the diffraction pattern originated by BPFLs at their focal planes. The simplicity of the method mainly relies on the small number of optical components needed for the calibration, which are limited to a common beam splitter, and an optical device able to measure light intensity changes. In fact, for optical layouts characterized by the use of reflective SLMs, having certain tilt with respect to the laser beam, the beam splitter for the calibration is also unnecessary. Hence, in many cases, the proposed method allows carrying out the calibration task without moving the SLM from the original position chosen for the application. In addition, due to its diffractivebased nature, the method is less affected by environmental changes than other calibration methods based on interferometric setups. For on-axis calibrations, the method generates focal irradiance distributions with circular symmetry, which can be convenient not only for phase calibration under quasi-monochromatic radiation, but also for simultaneously multi-wavelength calibration of broadband light sources by using an adequate spectrometer.

\section{REFERENCES}

C. Maurer, A. Jesacher, S. Bernet, and M. Ritsch-Marte, "What spatial light modulators can do for optical microscopy," Laser Photon. Rev., vol. 5, pp. 81-101 (2011).

Z. Kuang, W. Perrie, J. Leach, M. Sharp, S. P. Edwardson, M Padgett, G. Dearden, and K. G. Watkins, "High throughput diffractive multi-beam femtosecond laser processing using a spatial light modulator," Appl. Surf. Sci., vol. 255, pp. 2284-2289 (2008).

[3] G. Sinclair, J. Leach, P. Jordan, G. Gibson, E. Yao, Z. Laczik, M. Padgett, and J. Courtial, "Interactive application in holographic optical tweezers of a multi-plane Gerchberg-Saxton algorithm for three-dimensional light shaping,” Opt. Express, vol 12, pp. 16651670 (2004).

[4] J. Arines, V. Durán, Z. Jaroszewicz, J. Ares, E. Tajahuerce, P. Prado, J. Lancis, S. Bará, and V. Climent, "Measurement and compensation of optical aberrations using a single spatial light modulator," Opt. Express, vol. 15, pp. 15287-15292 (2007).

[5] A. M. Weiner and I. Introduction, "Femtosecond pulse shaping using spatial light modulators," Rev. Sci. Instrum., vol. 71, pp. 1929-1960 (2000).

[6] G. Lazarev, A. Hermerschmidt, S. Krüger, and S. Osten, "LCOS Spatial Light Modulators: Trends and Applications," in Optical Imaging and Metrology: Advanced Technologies, W. Osten and N. Reingand, eds (Wiley-VCH Verlag GmbH \& Co. KGaA 2012).

[7] N. Savage, "Digital spatial light modulators," Nature Photon., vol. 3, pp. 170-172 (2009).

[8] V. Durán, J. Lancis, E. Tajahuerce, and Z. Jaroszewicz, "Equivalent retarder-rotator approach to on-state twisted nematic liquid crystal displays", J. Appl. Phys., vol. 99, pp. 113101 (2006).

[9] J. A. Davis, I. Moreno, and P. Tsai, "Polarization eigenstates for twisted-nematic liquid-crystal displays", Appl. Opt., vol. 37, pp. 937-945 (1998)

[10] J. Andilla, E. Martín-Bardosa, S. Vallmitjana, "Prediction of phase-mostly modulation for holographic optical tweezers", Opt. Commun., vol. 281, pp. 3786-3791 (2008).

[11] P. Clemente, V. Duran, Ll. Martínez-León, V. Climent, E. Tajahuerce, and J. Lancis, "Use of polar decomposition of Muller 
matrices for optimizing the phase response of liquid-crystal-onsilicon display", Opt. Express., vol. 16, pp. 1965-1974 (2008).

[12] A. Bergeron, J. Gauvin, F. Gagnon, D. Gingras, H. H. Arsenault, and M. Doucet, "Phase calibration and applications of liquidcrystal spatial light modulator", Appl. Opt., vol. 34, pp. 5133-5139, 1995.

[13] X. Xun, and R. W. Cohn, "Phase calibration of nonuniform spatial light modulators”, Appl. Opt., vol. 35, pp. 6400-6406, 2004.

[14] S. Reichelt, "Spatially resolved phase-response calibration of liquid-crystal-based spatial light modulators", Appl. Opt., vol. 52, pp. 2610-2617, 2013.

[15] F. P. Ferreira, and M. S. Belsley, "Direct calibration of a spatial light modulator by lateral shearing interferometry", Opt. Express., vol. 18, pp. 7899-7904, 2010.

[16] D. Engstrom, G. Milewski, J. Bengtsson, and S. Galt, "Diffractionbased determination of the phase modulation for general spatial light modulators", Appl. Opt., vol. 45, pp. 7195-7204, 2006.

[17] Z. Zhang, G. Lu, F. S. Yu, "Simple method for measuring phase modulation in liquid crystal televisions", Opt. Eng., vol. 33, pp. 3018-3022, 1994.

[18] Ll. Martinez-León, Z. Jaroszewicz, A. Kolodziejczk, V. Durán, E. Tajahuerce, and J. Lancis "Phase calibration of spatial light modulators by means of Fresnel images", J. Opt. A: Pure Appl. Opt., vol. 11, pp. 125405, 2009.

[19] I. Moreno, P. Velásquez, C. R. Fernández-Pousa, M. M. SánchezLópez, and F. Mateos, "Jones matrix method for predicting and optimizing the optical modulation properties of a liquid-crystal display," J. Appl. Phys., vol. 94, pp. 3697-3702 (2003).

[20] A. Lizana, I. Moreno, A. Márquez, C. Iemmi, E. Fernandez, J. Campos, and M. J. Yzuel, "Time fluctuations of the phase modulation in a liquid crystal on silicon display: Characterization and effects in the diffractive optics", Opt. Express., vol. 16, pp. 16711-16722 (2008)

[21] J. L. Martínez, I. Moreno, M. Sanchéz-López, A. Vargas, and P. García-Martínez, "Analysis of multiple internal reflections in a parallel aligned liquid crystal on silicon SLM", Opt. Express., vol. 22, pp. 25866-25879 (2014).

[22] C. Lingel, T. Haist, and W. Osten, "Optimizing the diffraction efficiency of SLM-based holography with respect to the fringing field effect", Appl. Opt., vol. 52, 6877-6883 (2013).

[23] S. Sinzinger, and J. Jahns, Microoptics, 2nd, revised and enlarged edition, Germany: Wiley-VCH GmbH \& Co. KGaA Wiley, 2003, pp. $152-155$.

[24] V. Moreno, J. F. Román, and J. R. Salgueiro, "High efficiency diffractive lenses: Deduction of kinoform profile", Am. J. Phys., vol. 65, pp. 556-562, 1997.

[25] D. Engstrom, M. Persson, J. Bengtsson, and M. Goksor, "Calibration of spatial light modulators suffering from spatially varying phase response", Opt. Express., vol. 21, pp. 16086-16103, 2013. 


\section{List of figures}

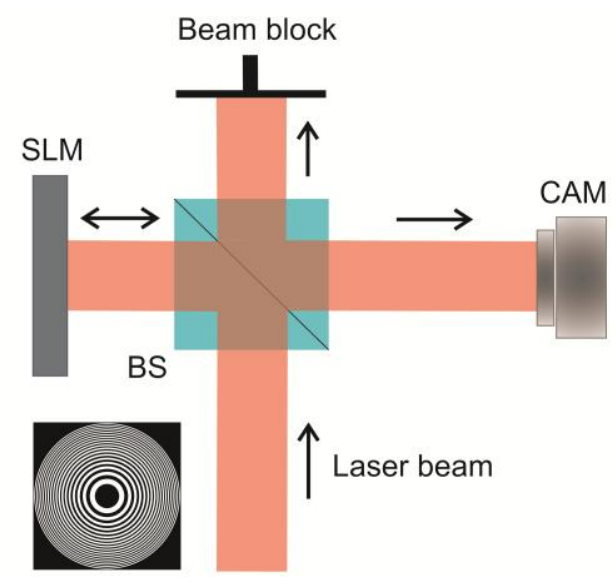

Figure 1. Optical setup used for phase calibration of a phase-only SLM. The light from a He-Ne laser impinges onto the SLM after being reflected in the beam splitter. The camera collected the diffracted light at the focal plane of BPFLs encoded into the SLM. 
(a)

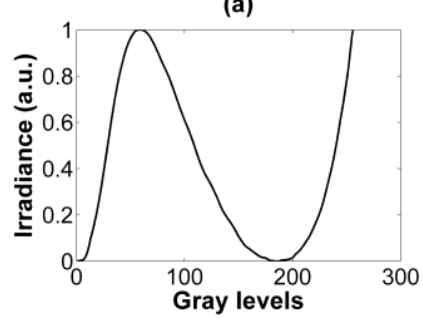

(b)

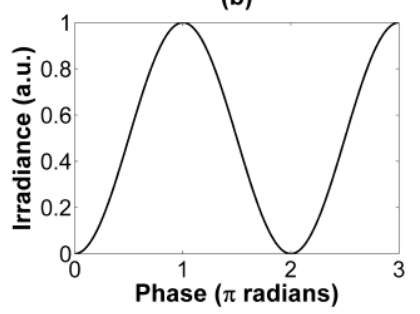

Figure 2. Integrated irradiance at main focus plane of BPFLs in the region defined by the $1 / e^{2}$ criterion, a) experiment and b) theoretical expression given in Eq. (2). 


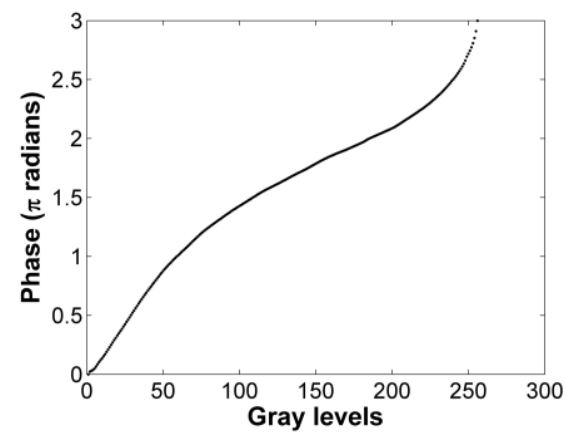

Figure 3. Phase calibration function obtained with the proposed method, which is based on a set of BPFLs encoded into the SLM. 


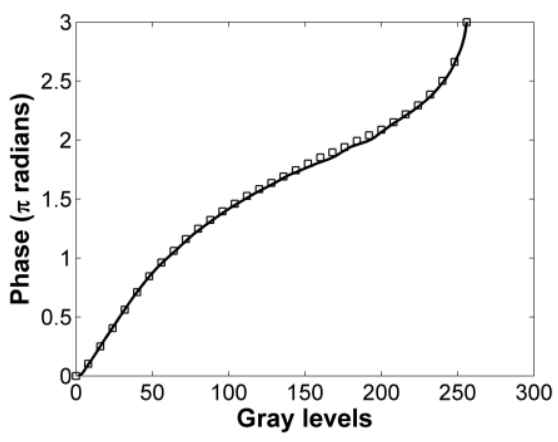

Figure 4. Comparison between two phase calibration functions, a) curve plotted with thick lines, obtained with the Ronchi grating method introduced in [17], b) curve plotted with square symbols, obtained with our proposed method using BPFLs. 


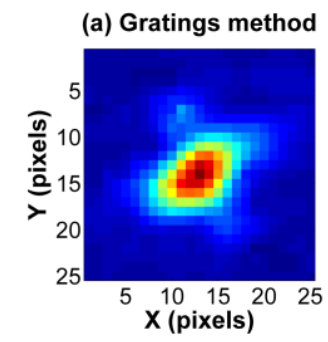

(b) BPFLs method

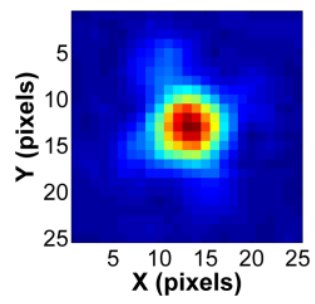

Figure 5. Focal irradiance distribution of a pair of foci used for phase calibration, a) with the method based on Ronchi gratings [17], b) with the method based on BPFLs proposed in this manuscript. 